DOI 10.35381/cm.v5i9.291

\title{
Evaluación formativa y la percepción del proyecto planificación curricular
}

\section{Formative evaluation and the perception of the curriculum planning project}

\author{
Evelyn Lizet Lugo Meza \\ tchuevlu@upc.pe \\ Universidad Peruana de Ciencias Aplicadas, Santiago de Surco \\ Perú \\ https://orcid.org/0000-0001-6137-8611
}

Recibido: 1 de mayo de 2019

Aprobado: 1 de junio de 2019

\section{RESUMEN}

La investigación se realizó con el objetivo de "determinar la relación entre el nivel de conocimiento de la evaluación formativa y la percepción del proyecto planificación curricular y evaluación formativa en docentes de instituciones educativas públicas de la Región Callao en el año 2018", con un diseño de campo no experimental. Fue de tipo correlacional, se trabajó con una muestra poblacional de 100 docentes a quienes se les aplicó dos instrumentos de medición tipo cuestionario con escalamiento de Likert de cinco alternativas de repuestas, estadísticamente se procesó mediante la correlación de Spearman. Al encontrarse una significancia bilateral de ,565 siendo mayor a 0.05 , se procede a aceptar la hipótesis nula $(\mathrm{H} 0)$. No existe relación entre el nivel de conocimiento de la evaluación formativa y la percepción del proyecto planificación curricular y evaluación formativa en docentes de Instituciones Educativas Públicas de la Región Callao en el año 2018.

Descriptores: planificación de la educación; innovación educacional; innovación pedagógica; investigación pedagógica.

\section{ABSTRACT}

The research was carried out with the objective of "determining the relationship between the level of knowledge of the formative evaluation and the perception of the curriculum 
planning project and formative evaluation in teachers of public educational institutions of the Callao Region in 2018", with a design non experimental field. It was correlational, with a population sample of 100 teachers who were applied two questionnaire measuring instruments with Likert scaling of five alternative answers, statistically processed by Spearman correlation. Upon finding a bilateral significance of, 565 being greater than 0.05 , the null hypothesis $(\mathrm{HO})$ is accepted. There is no relationship between the level of knowledge of the formative evaluation and the perception of the curriculum planning and formative evaluation project in teachers of Public Educational Institutions of the Callao Region in 2018.

Descriptors: educational planning, educational innovations, teaching method innovations, educational research.

\section{INTRODUCCIÓN}

En la actualidad, para desarrollar exitosamente sus actividades académicas (de enseñanza-aprendizaje) es necesario que los docentes conozcan dos términos que parecieran no ser relevantes, como lo son planificación y evaluación. En este contexto, todo proceso sistematizado, como lo es la enseñanza-aprendizaje, requiere de una planificación y evaluación, es decir de una previsión, realización y control de los diversos componentes que intervienen en dicho proceso.

La profesión docente está íntimamente ligada a la evaluación, por cuanto se realiza durante todo el proceso de formación, siguiendo muchas veces, criterios preestablecidos, lo cual conlleva a realizar las siguientes interrogantes ¿se evalúa bien? ¿Se adoptan los criterios correctos o adecuados? Siendo necesario conocer que la evaluación con carácter formativo no es lo mismo que calificar, medir o corregir, examinar, clasificar o aplicar pruebas objetivas. De ahí que Méndez citado por Grau (2010) sostiene que debe:

Entenderse como actividad crítica de aprendizaje, porque se asume que la evaluación es aprendizaje en el sentido que por ella adquirimos conocimiento. El profesor aprende para conocer y para mejorar la práctica docente en su complejidad, y para colaborar en el aprendizaje del alumnado conociendo las dificultades que tienen que superar, el modo de resolverlas y las estrategias que coloca en funcionamiento. El alumno aprende de y partir 
de la propia evaluación y de la corrección, de la información contrastada que le ofrece el profesorado, que será siempre crítica y argumentada, pero nunca descalificadora ni penalizadora. (p. 2)

Lo que conlleva a afrontar los cambios que requiere la educación del siglo XXI, se constituya en un reto muy importante y necesario. Este cambio que es preeminente en la forma de la docencia debe ser interactivo, según Grau (2010) se sustenta en tres principios: "a) mayor implicación y autonomía del alumnado, b) utilización de metodologías más activas y participativas, y c) papel del profesorado como agente sensibilizador y estimulador de los aprendizajes de los estudiantes" (p. 2). Los mejores resultados en el proceso de enseñanza-aprendizaje, no son adquiridos solamente con una buena planificación de contenidos de los cursos, de los objetivos de aprendizaje, si bien son importantes, pero no suficiente para el logro exitoso de los objetivos. Lo más importante radica en la planificación y aplicación de la evaluación como un proceso de cambio y mejora. Se comparte las palabras exactas de Méndez citado por Grau (2010) para referirse a la evaluación cuando dice que "evaluar es conocer, es contrastar, es dialogar, es indagar, es argumentar, es deliberar, es razonar, es aprender" (p. 3). Existen docentes que se cierran en la idea de que la evaluación es la aplicación de pruebas objetivas y con eso basta para saber si el estudiante "sabe o no sabe", es más, no toman en cuenta el proceso de evaluación, que como se sabe, tiene tres momentos esenciales, de inicio, de proceso o también llamada formativa y, la evaluación final; es decir, la evaluación se da durante todo el proceso.

Otro punto muy vinculado a la evaluación, es que los docentes conozcan la preeminencia de la planificación curricular en el desarrollo de las actividades académicas y su influencia en el logro de los objetivos planteados; entonces la pegunta es ahora ¿Qué es planificar? Para seguir la línea educativa, se cita al MINEDU (2017) que refiere al respecto:

Planificar es el arte de imaginar y diseñar procesos para que los estudiantes aprendan. La planificación es una hipótesis de trabajo, no es rígida, se basa en un diagnóstico de las necesidades de aprendizaje. En su proceso de 
ejecución, es posible hacer cambios en función de la evaluación que se haga del proceso de enseñanza y aprendizaje, con la finalidad de que sea más pertinente y eficaz al propósito de aprendizaje establecido (p. 4).

De todo lo planteado hasta ahora, se puede decir que planificar y evaluar son procesos estrechamente relacionados y se desarrollan de manera intrínseca al proceso de enseñanza-aprendizaje. La evaluación se considera como un proceso previo a la planificación permanente y al servicio de la mejora del aprendizaje durante el proceso de ejecución de lo planificado.

En síntesis, se puede señalar que la planificación y la evaluación deben ser dos elementos fundamentales de la labor docente, porque se tienen que asumir los retos y las exigencias de la educación del siglo XXI; debido a que ya no se quiere tener docentes improvisados, que desarrollan sus actividades académicas con el lema "a lo que salga" que aún creen que la evaluación consiste en la aplicación de pruebas objetivas. Por todo lo dicho, es que la presente investigación se enfoca en medir el nivel de conocimiento y la percepción de los docentes acerca de la planificación curricular y la evaluación formativa.

Los docentes en la actualidad, se enfrentan a una variada y difícil realidad en cada una de las instituciones educativas en las que se desempeñan, en las cuales se encuentran con cada estudiante que, a la vez, cada uno tiene diversos problemas, ya sea de aprendizaje, de conducta o de familia. Para a ello, el docente tiene que estar preparado y debe ser lo más creativo posible para poder afrontar y guiar a los estudiantes para que puedan superar cada uno de los problemas que los aqueja. Por ende, es importante que los docentes conozcan y apliquen la evaluación formativa en el desarrollo de sus actividades de enseñanza-aprendizaje, ya que esta le va permitir recabar información importante que le va ayudar a tomar decisiones, es por esta razón, además que el (MINEDU, 2017) con el apoyo de la Organización de las Naciones Unidas para la Educación, la Ciencia y la Cultura (UNESCO) implementaron en el 2017 un curso virtual con la finalidad de desarrollar competencias para la aplicación de los 
criterios propuestos por el Currículo Nacional de la Educación Básica, en el marco de los enfoques de la Planificación Curricular y Evaluación Formativa, que se supone contribuyeron en el mejoramiento de la práctica docente para el desarrollo de los aprendizajes de los estudiantes.

La metodología que se promovió para este curso se enmarcó en "el enfoque de la evaluación formativa, que permite al participante conocer desde un inicio lo que se espera logre al finalizar el curso, así como durante todo el proceso de desarrollo del curso en cada una de las actividades propuestas" (UPCH, 2017, pág. 5).

Esta metodología comprometía al tutor y participante para alcanzar los objetivos que perseguía el curso, es por esto que durante todo el proceso, el participante contó con el acompañamiento y orientación de su tutor quien iba monitoreando "el progreso alcanzado y las dificultades presentadas para que de manera conjunta aportar en su mejora" (UPCH, 2017, pág. 5). Asimismo, el participante recibía, de manera constante, la retroalimentación informada y oportuna para fortalecer, no solo el aspecto afectivo valorativo, sino que contribuya al manejo adecuado de los contenidos del curso.

Las actividades estuvieron orientadas a promover en los docentes participantes su capacidad reflexiva, de análisis, síntesis, argumentación, debate y planteamiento de conclusiones que orientaron la mejora de su práctica pedagógica. En el siguiente esquema se resume, de manera lúdica, la metodología del curso.

\section{MÉTODO}

La investigación siguió el enfoque cuantitativo de acuerdo a Hernández, Fernández \& Baptista (2014), con una tipología correlacional con la finalidad de cumplir con el objetivo de "determinar la relación entre el nivel de conocimiento de la evaluación formativa y la percepción del proyecto planificación curricular y evaluación formativa en docentes de instituciones educativas públicas de la Región Callao en el año 2018", con un diseño de campo no experimental.

Se trabajó con una muestra poblacional de 100 docentes a quienes se les aplicó dos 
instrumentos de medición tipo cuestionario con escalamiento de Likert de cinco alternativas de repuestas, validándose por el juicio de tres expertos y calculándose el coeficiente de confiabilidad de Alfa de Cronbach, para el instrumento 1: Nivel de conocimiento de evaluación formativa, se obtuvo alta confiabilidad de 0,901; mientras que para el instrumento 2: Percepción del proyecto planificación curricular y evaluación formativa, el nivel de confianza fue de 0,889 , estadísticamente se procesó mediante la correlación de Spearman.

\section{RESULTADOS}

\section{Cuadro 1}

Correlación entre el nivel de conocimiento de la evaluación formativa y la percepción del proyecto planificación curricular y evaluación formativa.

\begin{tabular}{|c|c|c|c|}
\hline & & $\begin{array}{l}\text { X: Percepción del } \\
\text { proyecto } \\
\text { planificación } \\
\text { curricular } \\
\text { evaluación } \\
\text { formativa. }\end{array}$ & $\begin{array}{l}\text { Y: Nivel de } \\
\text { conocimiento } \\
\text { de evaluación } \\
\text { formativa }\end{array}$ \\
\hline $\begin{array}{l}\text { X: Percepción del } \\
\text { proyecto planificación } \\
\text { curricular y evaluación } \\
\text { formativa. }\end{array}$ & $\begin{array}{l}\text { Correlación } \\
\text { de Pearson } \\
\text { Sig. } \\
\text { (bilateral) }\end{array}$ & 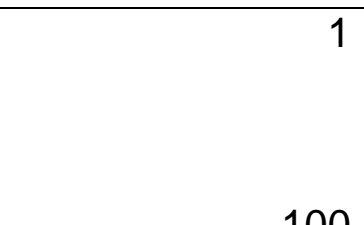 & $\begin{array}{l}, 058 \\
, 565\end{array}$ \\
\hline $\begin{array}{l}\text { Y: Nivel de conocimiento } \\
\text { de evaluación formativa }\end{array}$ & $\begin{array}{l}\mathrm{N} \\
\text { Correlación } \\
\text { de Pearson } \\
\text { Sig. } \\
\text { (bilateral) }\end{array}$ & $\begin{array}{l}100 \\
, 058 \\
, 565\end{array}$ & $\begin{array}{r}100 \\
1\end{array}$ \\
\hline
\end{tabular}

Al encontrarse una significancia bilateral de ,565 siendo mayor a 0.05 , se procede a aceptar la hipótesis nula $(\mathrm{H} 0)$ :

$\mathrm{H}_{0}$ : "El nivel de conocimiento de la evaluación formativa y la percepción del proyecto planificación curricular y evaluación formativa en docentes de Instituciones Educativas Públicas de la Región Callao en el año 2018 son independientes 
Por lo cual, se declara que ambas variables son independientes y no relacionan significativamente entre sí, lo cual incide que podría existir relación al azar o circunstancial durante el proceso de investigación, por cuanto existe una correlación positiva débil de 0,58 .

\section{DISCUSIÓN}

Los resultados obtenidos concuerdan con lo expuesto por Martínez Rizo (2013), al indicar que la evaluación formativa es una tendencia que requiere que el docente se encuentre empoderado del enfoque constructivista, con la finalidad de poder desarrollar las evaluaciones en concordancia con lo expresado por este modelo educativo, incluyendo que una de las actividades prioritarias del docente, es identificar la etapa de desarrollo cognitivo de los estudiantes con la finalidad de introducir nuevos conceptos y erradicar los preconceptos errados que puedan tener, siendo que el rol del docente se constituye en un asesor con fines de contribuir en el crecimiento integral del estudiante. Una medida que puede contribuir en la conformación de una nueva visión por parte del docente, se refiere a lo planteado por Canabal \& Margalef (2017), al indicar que es pertinente la configuración del investigador docente con la finalidad de propiciar un proceso de autorreflexión y retroalimentación con los estudiantes, mediante la aplicación de pruebas de tipo formativa, siendo necesario transcender el modelo mecanicista de transmisión de conocimientos, por el de uno en donde el estudiante genere mediante el auto aprendizaje, competencias que le permitan comprender la necesidad de aprender para la vida.

En complemento, Villasmil (2016) plantea que la autorreflexión es una estrategia fundamental para el docente como factor para transcender el modelo en donde el profesor es quien posee el conocimiento y el estudiante es un receptor pasivo, por consiguiente la reflexión es una evaluación que permite promover competencias intelectuales y de pensamiento crítico - reflexivo en los estudiantes, siendo necesaria su incorporación como un tipo de evaluación formativa. 
Así mismo, Hernández (2018), destaca la importancia de promover la participación de los padres y representantes en el proceso de aprendizaje de los estudiantes, así se puede ampliar el contorno de acción con el docente, aunado que el entorno cercano al estudiante tendría la oportunidad de participar en la articulación de un modelo educativo en donde se requiere de la cooperación de todos los actores educativos involucrados en la institución educativa.

\section{CONCLUSIONES}

No existe relación entre el nivel de conocimiento de la evaluación formativa y la percepción del proyecto planificación curricular y evaluación formativa en docentes de Instituciones Educativas Públicas de la Región Callao en el año 2018.

No existe relación entre la variable Nivel de conocimiento de la evaluación formativa y la dimensión Materiales de la variable Percepción del proyecto Planificación curricular y evaluación formativa en docentes de Instituciones Educativas Públicas de la Región Callao en el año 2018 con coeficientes de significancia bilateral 0,825 en el caso del coeficiente Pearson y 0,958 para Chi cuadrado.

No existe relación entre la variable Nivel de conocimiento de la evaluación formativa y la dimensión Accesibilidad de la variable Percepción del proyecto Planificación curricular y evaluación formativa en docentes de Instituciones Educativas Públicas de la Región Callao en el año 2018, con coeficientes de significancia bilateral 0,500 en el caso del coeficiente Pearson y 0,768 para Chi cuadrado.

No existe relación entre la variable Nivel de conocimiento de la evaluación formativa y la dimensión Tutoría de la variable Percepción del proyecto Planificación curricular y evaluación formativa en docentes de Instituciones Educativas Públicas de la Región Callao en el año 2018, con coeficientes de significancia bilateral 0,227 en el caso del coeficiente Pearson y 0,383 para Chi cuadrado.

No existe relación entre la variable Nivel de conocimiento de la evaluación formativa y la dimensión Interactividad de la variable Percepción del proyecto Planificación curricular y 
evaluación formativa en docentes de Instituciones Educativas Públicas de la Región Callao en el año 2018, con coeficientes de significancia bilateral 0,227 en el caso del coeficiente Pearson y 0,383 para Chi cuadrado.

No existe una relación entre la variable Nivel de conocimiento de la evaluación formativa y la dimensión Interacción de la variable Percepción del proyecto Planificación curricular y evaluación formativa en docentes de instituciones educativas públicas de la Región Callao en el año 2018, con coeficientes de significancia bilateral 0,227 en el caso del coeficiente Pearson y 0,383 para Chi cuadrado.

La variable X: Percepción del proyecto planificación curricular y evaluación formativa tiene un desarrollo más homogéneo que la variable $Y$ : Nivel de conocimiento de evaluación formativa.

El proyecto no tiene un impacto relevante ni significativo en los docentes de instituciones educativas públicas de la Región Callao en el año 2018.

La dimensión con desarrollo más heterogéneo de la variable Y: Nivel de conocimiento de evaluación formativa es la Y1: Comprensión básica, por otro lado, también la dimensión con un desarrollo más homogéneo es Y4: Retroalimentación.

Sobre la variable $\mathrm{X}$ : Percepción del proyecto planificación curricular y evaluación formativa, su dimensión con más homogenización X3: Tutoría, y con más heterogeneidad es la dimensión X5: Interacción.

\section{REFERENCIAS CONSULTADAS}

1. Canabal, C., \& Margalef, L. (2017). La retroalimentación: la clave para una evaluación orientada al aprendizaje. Profesorado, Revista De Curriculum Y Formación Del Profesorado, 21(2), 149-170. doi:10.30827/profesorado.v21i2.10329

2. Grau C., S. (2010). La evaluación un proceso de cambio para el aprendizaje. España: Universidad de Alicante.

3. Hernández, A. (2018). Transitando por el camino de la escuela para padres, madres y representantes. Una experiencia vivida. EPISTEME KOINONIA, 1(1), 
$51-71$.

https://fundacionkoinonia.com.ve/ojs/index.php/epistemekoinonia/article/view/490

4. Hernández, R., Fernández, C., Baptista, P. (2014). Metodología de la Investigación. México: Editorial Mc - Graw - Hill Interamericana.

5. Martínez Rizo, Felipe. (2013). Dificultades para implementar la evaluación formativa: Revisión de literatura. Perfiles educativos, 35(139), 128-150. Recuperado a partir de http://www.scielo.org.mx/scielo.php?script=sci_arttext\&pid=S0185$26982013000100009 \& \operatorname{lng}=e s \&$ tIng=es.

6. MINEDU. (2017). Cartilla de planificación Curricular. Lima: MINEDU.

7. UPCH. (2017). Planificación Curricular y Evaluación Formativa. Lima: UNIVERSIDAD PERUANA CAYETANO HEREDIA.

8. Villasmil Y., D. (2016). La reflexión como factor fundamental de la praxis pedagógica del docente universitario. Revista Arbitrada Interdisciplinaria Koinonía, 1(2), 103-122. Recuperado de https://fundacionkoinonia.com.ve/ojs/index.php/revistakoinonia/article/view/41/28

\section{REFERENCES CONSULTED}

1. Canabal, C., \& Margalef, L. (2017). Feedback: the key to a learning oriented evaluation. Teachers, Journal of Curriculum and Teacher Training, 21 (2), 149170. doi: 10.30827 / profesorado.v21i2.10329

2. Grau C., S. (2010). Evaluation a process of change for learning. Spain: University of Alicante.

3. Hernández, A. (2018). Transiting the school path for fathers, mothers and representatives. A lived experience. EPISTEME KOINONIA, 1 (1), 51-71. Recovered https://fundacionkoinonia.com.ve/ojs/index.php/epistemekoinonia/article/view/490

4. Hernández, R., Fernández, C., Baptista, P. (2014). Investigation methodology. Mexico: Editorial Mc - Graw - Interamerican Hill.

5. Martínez Rizo, Felipe. (2013). Difficulties to implement the formative evaluation: 
Literature review. Educational profiles, 35 (139), 128-150. Retrieved from http://www.scielo.org.mx/scielo.php?script=sci_arttext\&pid=S0185$26982013000100009 \&$ Ing=en\&tIng=en.

6. MINEDU (2017). Curriculum planning chart. Lima: MINEDU.

7. UPCH (2017). Curriculum Planning and Formative Evaluation. Lima: PERUANA CAYETANO HEREDIA UNIVERSITY.

8. Villasmil Y., D. (2016). Reflection as a fundamental factor in the pedagogical praxis of the university teacher. Interdisciplinary Arbitrated Review Koinonía, 1 (2), 103-122. Recovered from https://fundacionkoinonia.com.ve/ojs/index.php/revistakoinonia/article/view/41/28 